
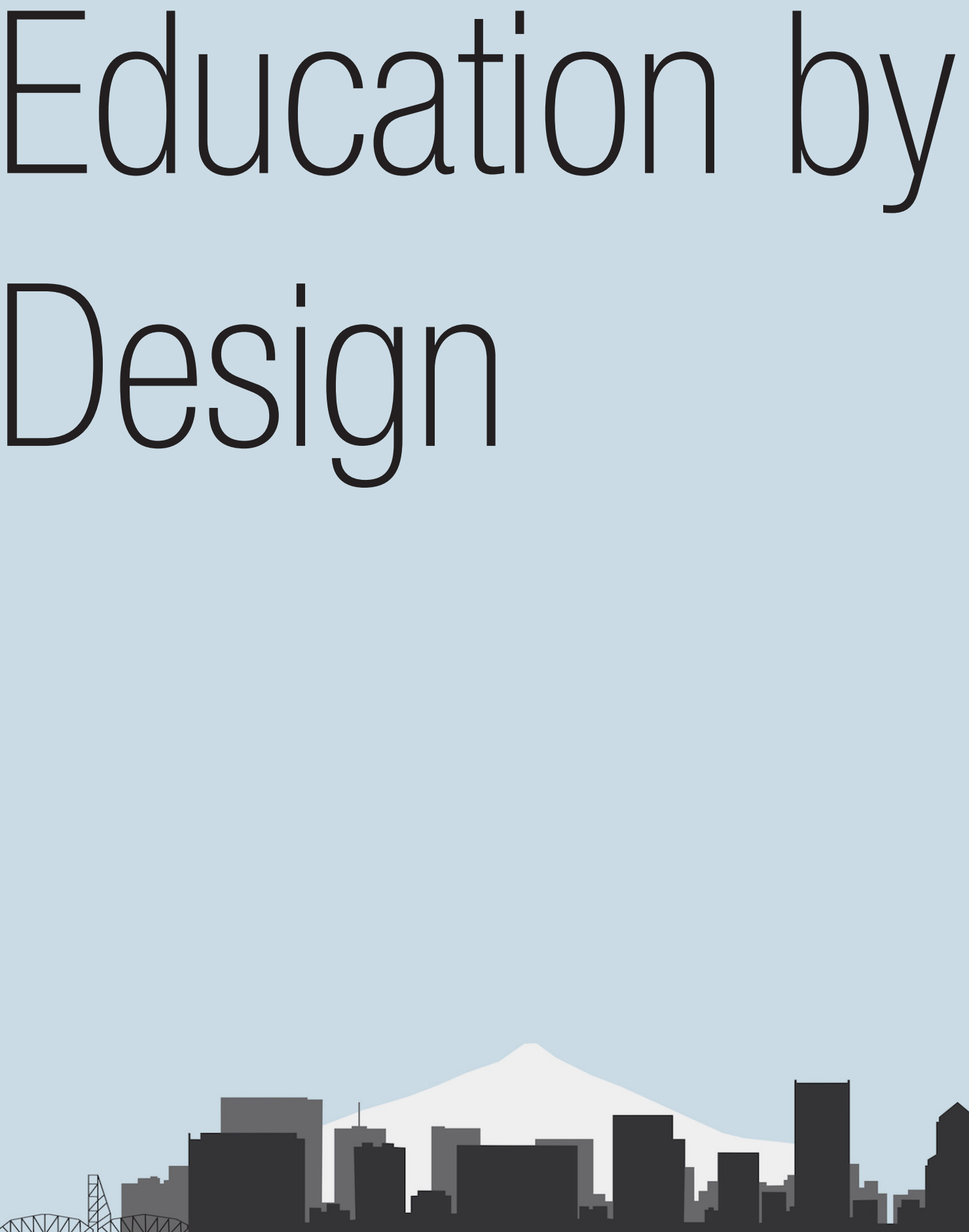

2019 Reynolds Symposium: Education by Design October 18-20, 2019 


\section{Organizing Committee:}

[co-organizers]

Professor Alison Kwok, Ph.D, FAIA, University of Oregon

Emeritus Professor John Reynolds, FAIA, University of Oregon

\section{[Symposium coordinator]}

Isabel Rivera, Ph.D., University of Oregon

Professor Walter Grondzik, P.E., Ball State University

Professor Bruce Haglund, AIA Assoc., University of Idaho

Assistant Professor Emily McGlohn, AIA, Auburn University

Associate Professor Ulrike Passe, lowa State University

Assistant Professor Siobhan Rockcastle, Ph.D., University of Oregon

Sharon Refvem, FAIA, LEED Fellow, Senior Associate and Director, Sustainability Resource Group, Hawley Peterson Snyder 


\title{
Mbesese Build: An Experimental Experience
}

\author{
Kevin Dong \\ Professor \\ Cal Poly - SLO \\ San Luis Obispo, CA \\ kdong@calpoly.edu
}

\author{
Thomas Fowler \\ Professor \\ Cal Poly - SLO \\ San Luis Obispo, CA \\ tfowler@calpoly.edu
}

\begin{abstract}
The Same' Polytechnic College is a proposed vocational training institution in the United Republic of Tanzania to provide educational opportunities which increase human capital, with the goal of reducing severe levels of poverty. The college is the pilot project for the Mbesese Initiative for Sustainable Design (MISD). MISD has partnered with design firms and the university to develop a framework for campus development. The university team established overarching planning principles for buildings and related infrastructure to support 1,200 students.

The project provided a platform for collaboration between faculty, students, and design professionals. The campus proposal encompasses architecture, planning, and a variety of engineering disciplines. Students researched topics that are requisite to building; energy usage and generation, water conservation and reclamation, natural ventilation and thermal comfort, day lighting and solar exposure, construction materials and structural systems, pedestrian and vehicular traffic patterns, and, site access and maintenance. The masterplan recommendations are based on computational analysis and design, results from laboratory experiments, and feedback from design professionals. The students then developed building strategies for implementing the aforementioned concepts, while learning how those design issues are intertwined.

In 2018, students, faculty, and MISD volunteers constructed a micro structure in Tanzania based on the master plan recommendations and results from laboratory experiments. The building process allowed the team to better understand how cultural, environmental, and technological considerations influence design and building in developing areas. The linkage between experimental research, design, and construction is a hallmark for the project both at the university and in Tanzania.
\end{abstract}

\section{STUDIO: THE COLLABORATORY}

\section{The studio philosophy}

The goal of the academy is to educate future design professionals, prepare individuals for a changing world, and provoke thoughtful designs which address environmental and performance criteria while exhibiting technical excellence. In order to do this, the authors have developed a studio which blurs the line between structures and architecture using the philosophies embodied in Ove Arup's Key Speech in which he describes the melding of disciplines to create a holistic design; and studio-based learning outlined in Donald Schon's work, Educating the Reflective Practitioner (1987) in which he states: "Designing, both in its narrower architectural sense and in the broader sense in which all professional practice is design like, must be learned by doing.

\section{Functioning Teams}

The class or "Collaboratory" is similar to other interdisciplinary studios offered within the college with students from structures and architecture. But the authors promote a round table approach to design - no one person is at the head of the table, but at different phases of design, different individuals take the lead. Additionally, a team-based approach to problem solving is stressed to signify the importance of working in groups, reinforce the benefits of diverse perspectives, and to highlight the intersections between 
disciplines. Students are required to read and reflect on selected chapters from books by Balmond (2010) and Olson \& McNamara (2014) which discuss the relationships between design professionals and alternative approaches to meld structures and architecture. A key objective for the course is to understand the characteristics necessary for strong interdisciplinary collaboration. (Dong, Doerfler 2013)

Intermediate benchmarks are assessed twice weekly with desk critiques or "crits" and short interdisciplinary presentations. All team members are expected to contribute to the conversation to ensure the team is working towards the same goal and all members understand the 'core values' of the design. Occasionally, the roles are switched when the architecture students describe structure and the structural engineering students describe the architecture and material palette. This is a critical piece to the course. The role reversal identifies when members do and don't understand how their discipline is intertwined with others. The frequent crits emulate real world practice in that consultants are continually coordinating and integrating the work of others and benchmarking progress based on drawings. The instructors gauge team success on technical merit, but also peer evaluations which utilize a "trust battery" model for assessing teamwork, see Figure 1 below. Frequently the battery rating, one of three metrics used for evaluation, often mimics the instructor's observations and when one or two individuals tend to control the desk crits.

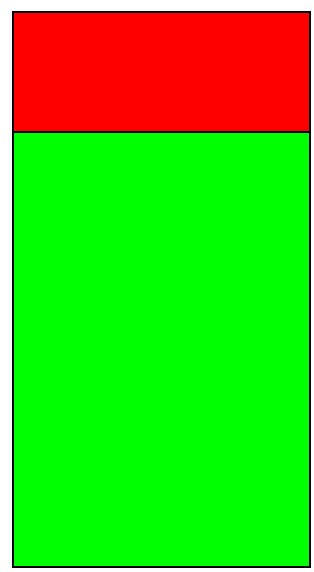

\section{INDICATE YOUR TRUST BATTERY RATING FOR YOUR TEAM WORK?}

Is it at 50\% (average); 30\% (needs work), or 80\% (in good shape but could use some adjustments):

TEAM TRUST BATTERY PRECENTAGE: $90 \%$ INDENTIFY THE TOP 1-2 THINGS TO WORK ON TO INCREASE THE “TRUST BATTERY” PERCENTAGE OF YOUR TEAM:

1. Improve organization. 2. Improve quality of deliverables. INDICATE YOUR “TRUST BATTERY” RATING FOR THE FIRST SURVEY HERE: $80 \%$

"The trust battery percentage has improved during the $2^{\text {nd }}$ survey because each of us have better understanding of the strengths and weaknesses of every team member."

Figure 1. Powering a Team With a "Trust Battery", Tobi Lütke.

The success of the team, an individual, or the project proposal is vetted through internal reviews, but more importantly through design professional critiques and presentations to users and owners. Student teams interview owners and users plus participate in engineering workshops, so teams are expected to incorporate that input and demonstrate how it is addressed during presentations. The process holds teams accountable and makes client and workshop input relevant. When real world projects are used for teaching, the opinions and comments from owners, users, and experienced professionals is vital to the success of a project and the Collaboratory tries to simulate this aspect. The successful student designs which address the owners brief or solve engineering challenges, move on to the next stage. For example, student designs for a greenhouse are being used for fund raising at a marine biology research center and drawings for the Same Polytechnic test building are used for construction, as shown in Figures 2 and 3 on the next page.

\section{The Current Mode}

Over the past three years, the instructors have taken the Collaboratory model a step further and have begun to blur the lines between academia and practice. The studio is part of a team which includes the philanthropic arm of a global engineering firm, Arup, a non-profit organization, MISD, working in the United States and in Tanzania, Africa. In addition to defining the campus master plan and developing building designs, the studio is engaged in research, experimentation, and on-site construction. Students from structures, mechanical, and electrical engineering have engaged in research to develop strength and fabrication recommendations, quantify natural ventilation effects and heat gain within classroom buildings (Hoang, Nute, Smith 2018), and propose the best mix of renewable and grid provided energy for 
cost and reliability (Hua 2018). The research findings and experimental results help inform both the students and the design professionals working on the project, such that more holistic solutions are proposed and indigenous building materials may be used in innovative ways.

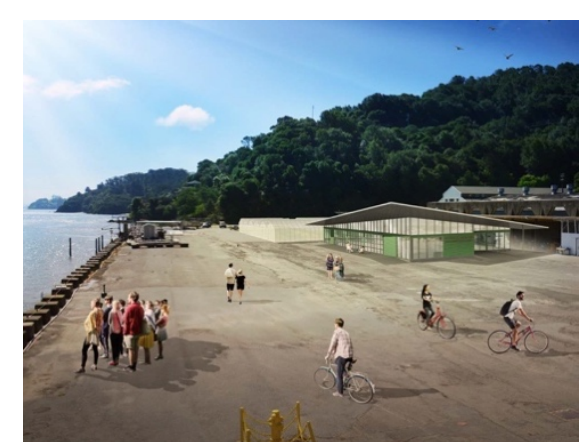

Figure 2. The Greenhouse Design at CSU

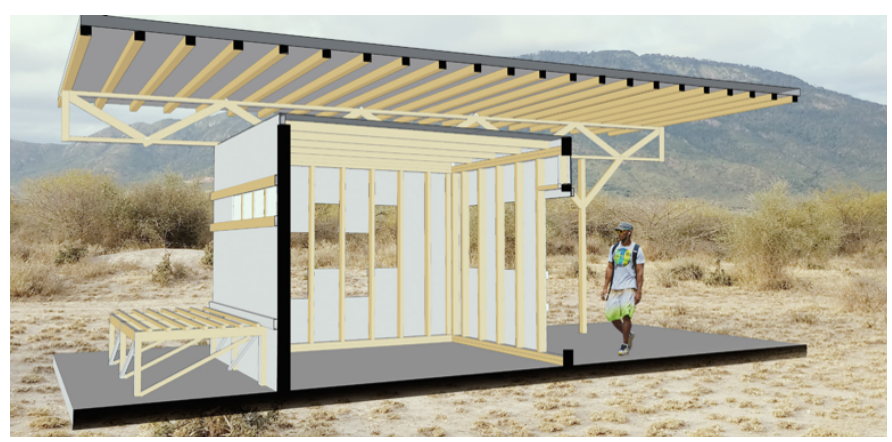

Figure 3. The Same Polytechnic Test Structure Design

\section{THE SAME POLYTECHNIC}

\section{The Tanzania back drop}

In 2012, the studio was invited to partner with a non-profit organization to aide in the development of a technical college in Same (pronounced Sah-may), Tanzania. The studio serves as the think tank for innovative solutions and out of the box ideas for shifting building design strategies. The students collaborated with a global engineering firm to hone building designs and incorporate feedback from practitioners who help mentor the students. The groups participate in a variety of workshops to learn about construction in developing countries, identify key design concepts that address the non-profit's goals, and how socio and economic considerations influence building design. (Dong, Fowler 2016)

Over the years, the project scope has broadened and the building designs have become more detailed. This is a natural progression with time, but the primary reason is the experience gained by visiting the site and spending time in the Tanzania. Initial proposals included individual buildings and a master plan based on research or shared information from the non-profit client, MISD (Dong, Fowler 2016). Since March 2015, the authors have visited Tanzania on eight separate occasions with construction occurring in 2017 and 2018. This is a key aspect to the design progression; it allowed students, instructors, and volunteers the ability to connect design with materials and construction. It also enabled the next iterations to translate that information into more realistic and integrated proposals. The knowledge gained from viewing the site, learning about the culture, and being immersed in the local environment was invaluable. As noted previously, it has led to studies regarding power sources, material property investigations, thermal comfort studies, and wind tunnel laboratory testing.

\section{Discover Tanzania!}

For the past three years, groups have travelled to Same and become acquainted with local culture, and environmental conditions, but also to evaluate building practices and better understand material availability. This has resulted in a number of research projects which involve material testing, structural systems modelling, and environmental design concepts for water collection and sustainability. The findings from these quantitative projects have informed the massing and orientations for the campus buildings, zoning and adjacencies for public and private areas, and are now incorporated into the design principles for the master plan (See Figures 4 and 5 below)

In the past year, student research has enabled the design team to better understand the material properties of cement blocks that are manufactured ubiquitously in Tanzania. A group of architectural engineering students replicated the mix design used for blocks in Tanzania to quantify the material properties and to develop design parameters for the flexural capacity of block walls subjected to out of 
plane loading. That work is on-going and two test sets have been completed. The student investigators have established strength and stiffness properties for the blocks, and are developing a predictive model for various block wall patterns.

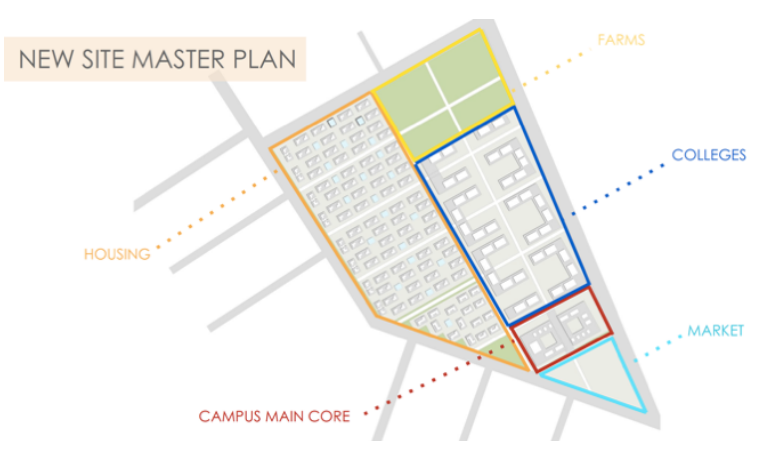

Figure 4. Schematic master plan

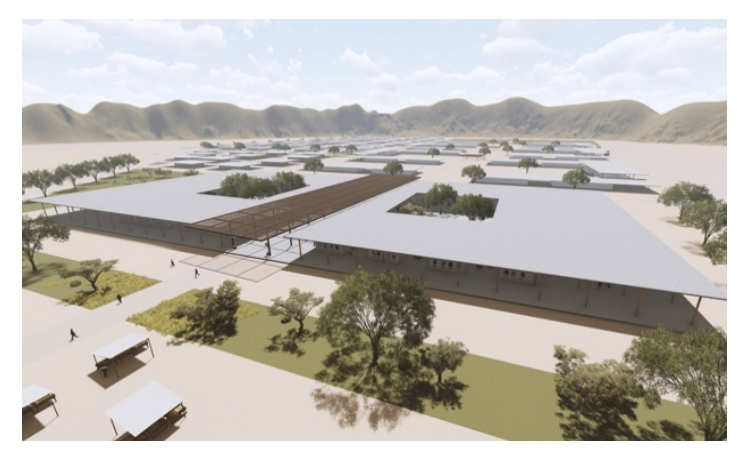

Figure 5. Proposed campus buildings

Concurrently with the structural block testing, a student group measured the thermal conductivity of the blocks and used that data to simulate thermal heat gains in the proposed buildings. The studies reinforced the reasoning for the "double" roof configuration shown in Figure 8 below, but study also showed that slight modifications to the roof composition would reduce radiant heat below. (Hoang, Nute, Smith 2018).

A third study was completed by the same group which conducted wind tunnel testing in order to measure air flow within typical classroom and office spaces. The architecture students used rules of thumb, provided by MISD design professionals, to position and determine wall or window opening sizes and locations (Figure 6). The tests were conducted to validate the opening placements or suggest modifications to enhance natural ventilation in the spaces. The testing, as shown in Figure 7, showed adequate ventilation across the space for moderate wind speeds, but also showed how slight modifications to the opening sizes or locations would improve ventilation at "dead spots" within the building. The architecture students used this information to refine the design and then the structures students verified the opening locations worked for strength. The architectural elevation is shown in Figure 8.

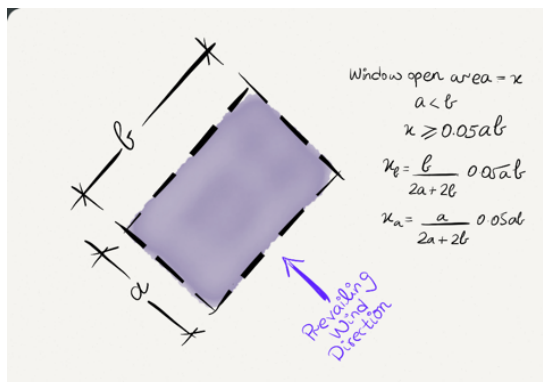

Figure 6. Rules of thumb for openings

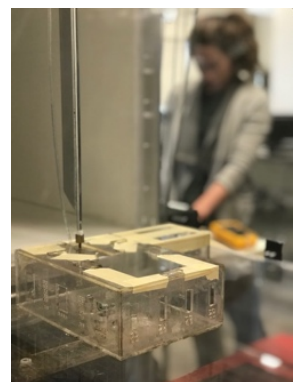

Figure 7. wind tunnel testing

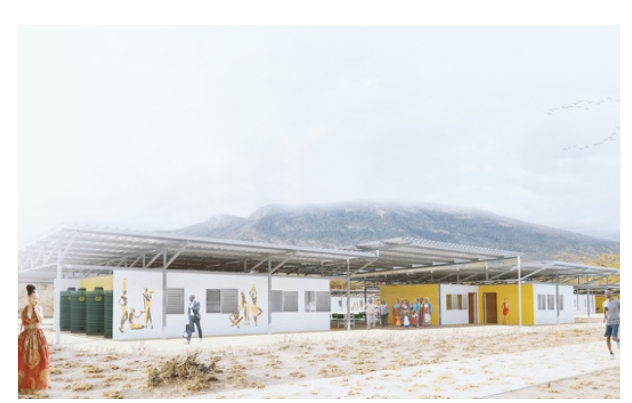

Figure 8. Architectural Elevation

\section{Build Tanzania}

The testing and research re-affirmed the groups decision to create buildings with "double" roofs as shown in Figure 5 and Figure 8. This type of construction is not common in Tanzania, but there are a few precedent buildings. The studies showed that the interior heat gains were dramatically decreased with this configuration due to the reduction in direct solar radiation. Additionally, based on lab testing, the concrete blocks demonstrated a low compressive strength, so the structural design utilized the blocks as non-load bearing exterior walls with the roof supported by steel post and beam construction.

Using the research data from the laboratory tests, and information gathered from site visits, a simple test structure was designed and built in the summer of 2018. The goal was to determine best practices for 
building in Tanzania and provide a prototypical structure for monitoring wind, rain, and interior temperature to validate the assumptions made in studio.

The team worked with local laborers to understand local practices, and purchased all materials from local vendors so, a cost model for future construction could be established. Another goal was Learn by Doing; demonstrating the relationship between drawings and construction, the impacts of building tolerances, and how to apply building techniques and engineering theory into practice. The images in Figure 9 show the model built in class and the process the team used to construct the final structure which incorporated concrete, steel, and wood.
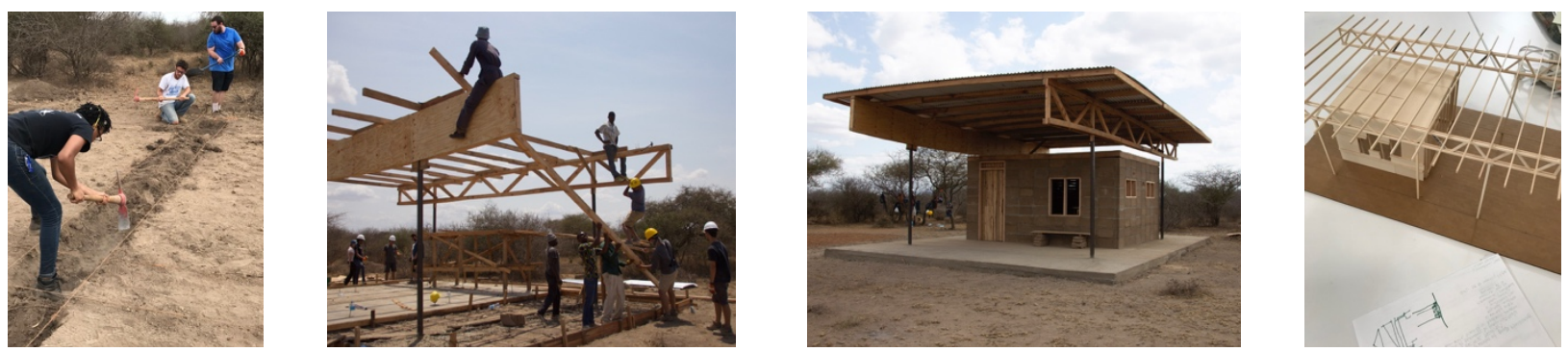

Figure 9. Trenching for grade beams; roof framing; finished structure; structural model made at University

The group learned how structures are made using manual techniques and geometry. The foundation was squared using twine, a tape measure, and the Pythagorean Theorem; the foundation was excavated with picks, shovels, and wheel barrows; concrete was mixed on the ground using shovels, "pumped" to site in wheel barrows, and levelled with a "2x4" and trowels; and framing was plumbed using a level and three ropes to form a tripod, etc... These experiences are invaluable. The students become informed designers, not only for the polytechnic college, but in preparation for their careers. They now consider challenges and considerations related to culture, material availability, building technology and accessibility, and how geometry is integrated into construction.

\section{Lessons Learned}

The studio, comprised primarily of structural engineering and architecture students, continues to develop the designs for the campus. In the latest studio iteration, the student teams incorporated results from tests completed by mechanical engineering students and worked with an electrical engineering student to include solar energy requirements, such as a solar farm. The inclusion of this information has resulted in holistic designs and buildings which portray a more accurate representation of the future buildings.

The University's motto is "Learn By Doing" which places an emphasis on making or which exemplify the application of theory to real world problems. During the past two years, student teams designed, then built their design in a developing area, and learned how diverse cultures inform design making decisions. Participants become aware of social and economic issues that are not common in most industrialized countries, then they learn how structures and architecture can respond to the needs of a developing country. At our school, the opportunity for students to use their gifts, work abroad, and contribute to society resonates with our student body. Projects like the Same Polytechnic are not uncommon, but the authors believe the approach, the process, and the experience are unique. Enabling students to participate in service learning projects is impactful and is reflected in the student comments included below;

"I traveled to Tanzania with vague expectations to learn about culture and construction. However, I left Tanzania with much more: a tremendous appreciation for people I never knew, lifestyles I didn't know existed, labor I hadn't realized was necessary, and a country which has impacted my career and life path." 
"Traveling and working throughout Tanzania last summer was an amazing experience. I've never felt so welcomed into a community so far abroad before. The lessons I learned from people, culture and construction will last a lifetime."

"At first, I was really excited about the idea of working on construction and design abroad in Africa. However, after spending a few days in Tanzania, I began to fall in love with the community surrounding the work we were doing. Traveling and working throughout Tanzania gave me so many opportunities to learn about different aspects of culture and construction. The lessons I learned from this trip could not have been experienced at my desk in school."

\section{CLOSING THOUGHTS}

\section{Conclusions}

The success of the Collaboratory is three-fold. First is availability of projects which require multiple disciplines to create holistic solutions. This allows the student teams to emulate associations across professions similar to the real world. The second is the availability of projects with longer project deadlines. The instructors work with the "clients" to determine the appropriate scope and more importantly schedule. The academic schedule is driven by factors much different than private sector schedules, so negotiating phased deliveries and identifying which service match well with student skill sets is imperative. The authors are fortunate to work with clients also in the non-profit world where time constraints are more similar to that in academia. And lastly, the success is based on the teamwork. The willingness of design professionals, owners, and users greatly enhances the learning experience for the students and the instructors. Similarly, they comment that working with students has broadened their horizons and they've gained an appreciation for the student's innovative ideas and their energy and passion for the project.

And lastly, the authors enjoy working together and is a primary reason they have co-taught this studio for almost ten years. They admire each other's work, value each other's ideas, and respect each other's contributions. This chemistry has allowed them to freely share ideas with each other, but more importantly with students, design professionals, and colleagues .

\section{Acknowledgements}

The authors thank our teammates, whose belief in our studio and willingness to work with students has provided an invaluable platform for teaching, mentoring, and preparing future leaders for the built environment.

A special thanks to John Kern, Associate Director with the Romberg Tiburon Center in Sausalito, California, a marine biology research facility for San Francisco State University. He provided one of the first explorations for the Collaboratory. His insights into land use history, exposure to researchers/users, and patience enabled the authors to identify how students work best with clients and on which type of projects. The studio has contributed to the preservation of a theater and developed collateral material for a headhouse facility on-site. In return students learned about sea level rise and how structural engineering is relevant at the biological level

We are indebted to the volunteers working with the Mbesese Initiative for Sustainable Design (MISD) and the design professionals who volunteer their time and expertise. The designs are holistic and the social aspects of Tanzanian life are incorporated into the proposals because of David Lambert, Tarrah Beebe, and Mansuetus Setonga. The students have gained an appreciation for cultures in developing areas, learned to design with purpose, and experienced design-build first hand.

And lastly, thank you to our Collaborative Workshop leaders Paul Switenki (Arup), Jonas Houston (Holmes), and Steve Slovek (HOK). Your insights and thoughtful dialog with students broadened their horizons when discovering the nuances of team work, the demands of leadership, and the art of negotiation. 


\section{REFERENCES}

Arup Journal, The Key Speech, Ove Arup and Partners, London, 1986

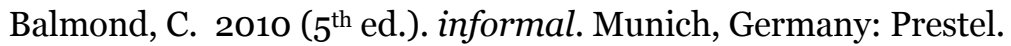

Dong, K., Doerfler, J., Fowler, T. “The Interdisciplinary Design Studio - Identifying Collaboration”, International Conference of Structures and Architecture, $2^{\text {nd }}$ Conference Proceedings, ICSA, Guimarães, Portugal, July 2013

Olsen, C. and Mac Namara, S. 2014. Collaborations in Architecture and Engineering, New York, NY: Routledge.

Dong, K., Fowler, T., "Crossing Boundaries: Blurring the Lines Between Engineers and Architects", International Conference of Structures and Architecture, $3^{\text {rd }}$ Conference Proceedings, ICSA, Guimarães, Portugal, July 2016.

Hua, K., Designing a Power System in Tanzania, Africa: The Load Profile, Power System Design, Design Simulation, and Cost Analysis for the Same polytechnic College, Digital Commons, San Luis Obispo, CA, June 2018.

Hoang, L., Nute, K., Smith, M., Same Polytechnic College, Mechanical Team: Final Design Report, Digital Commons, San Luis Obispo, CA June 2018 